\title{
Endogenous Angiogenesis Inhibitor Vasohibin1 Exhibits Broad-Spectrum Antilymphangiogenic Activity and Suppresses Lymph Node Metastasis
}

\author{
Takahiro Heishi, ${ }^{*}$ Tomoko Hosaka, ${ }^{*}$ \\ Yasuhiro Suzuki, ${ }^{*}$ Hiroki Miyashita, ${ }^{*}$ Yuichi Oike, ${ }^{\dagger}$ \\ Takashi Takahashi, ${ }^{\ddagger}$ Takumi Nakamura, ${ }^{\S}$ \\ Shingo Arioka, ${ }^{\S}$ Yuichi Mitsuda, ${ }^{\S}$ \\ Tomoaki Takakura, ${ }^{\S}$ Kanji Hojo, ${ }^{\S}$ \\ Mitsunobu Matsumoto, ${ }^{\S}$ Chihiro Yamauchi, ${ }^{\S}$ \\ Hideki Ohta, ${ }^{\S}$ Hikaru Sonoda, ${ }^{\S}$ \\ and Yasufumi Sato*

\begin{abstract}
From the Department of Vascular Biology,* Institute of Development, Aging, and Cancer, Tohoku University, Sendai; the Sciences, Kumamoto University, Kumamoto; the Division of Molecular Carcinogenesis, ${ }^{*}$ Center for Neurological Diseases and Cancer, Nagoya University Graduate School of Medicine,
\end{abstract} \\ Department of Molecular Genetics, ${ }^{\dagger}$ Graduate School of Medical \\ Nagoya; and the Discovery Research Laboratories, ${ }^{\S}$ Shionogi $\&$ \\ Co. Ltd., Osaka, Japan
}

During cancer progression, the angiogenesis that occurs is involved in tumor growth and hematogenousdistant metastasis, whereas lymphangiogenesis is involved in regional lymph node metastasis. Angiogenesis is counterregulated by various endogenous inhibitors; however, little is known about endogenous inhibitors of lymphangiogenesis. We recently isolated vasohibin1 as an angiogenesis inhibitor intrinsic to the endothelium and further demonstrated its anticancer activity through angiogenesis inhibition. Here, we examined the effect of vasohibin 1 on lymphangiogenesis. Vasohibin1 exhibited broad-spectrum antilymphangiogenic activity in the mouse cornea induced by factors including VEGF-A, VEGF-C, FGF2, and PDGF-BB. We then inoculated highly lymph node-metastatic cancer cells into mice and examined the effect of vasohibin1 on lymph node metastasis. Tail-vein injection of adenovirus containing the human vasobibin1 gene inhibited tumor lymphangiogenesis and regional lymph node metastasis. Moreover, local injection of recombinant vasohibin1 inhibited lymph node metastasis. These results suggest vasohibin1 to be the first known intrinsic factor having broad-spectrum antilymphangiogenic activity and indicate that it suppresses lymph node metastasis. (Am J Pathol 2010, 176:1950-1958; DOI: 10.2353/ajpath.2010.090829)

Peripheral lymphatic vessels, which are composed of a single layer of lymphatic endothelial cells (LECS) without mural cell coverage, collect fluid lost from blood vessels and maintain immune responses, lipid uptake, and tissue homeostasis. ${ }^{1}$ Recently, attention has focused on lymphangiogenesis, which is the formation of new lymphatic vessels, because it has been shown to be related to lymph node (LN) metastasis of cancers. ${ }^{2}$ Metastasis of malignant tumors to regional LNs is one of the early signs of spreading cancer, and it occurs as frequently as hematogenous distant metastasis. ${ }^{3}$

The formation of blood and lymphatic vessels is primarily controlled by vascular endothelial growth factor (VEGF) family members. ${ }^{4}$ This family of growth factors consists of 5 members (ie, VEGF-A, VEGF-B, VEGF-C, VEGF-D, and placental growth factor). There are also 3 types of VEGF receptor (VEGFR) tyrosine kinases: VEGFR1, VEGFR2, and VEGFR3. The most important molecule in the VEGF family that mediates angiogenesis of the formation of new blood vessels is VEGF-A, and VEGFR2 is the major mediator of VEGF-A-driven responses in blood endothelial cells (BECs). Alternatively, the most important factors that mediate lymphangiogenesis are VEGF-C and VEGF-D, and VEGFR3 is the major mediator of VEGF-C- and VEGF-Dmediated responses in LECs. ${ }^{4}$ In addition, several factors such as fibroblast growth factor (FGF)2, platele-derived growth factor BB (PDGF-BB), insulin-like growth factor 1

Supported by a grant from the program Grants-in-Aid for Scientific Research on Priority Areas from the Japanese Ministry of Education, Science, Sports, and Culture; by Health and Labor Sciences research grants; and by funding from Third Term Comprehensive Control Research for Cancer from the Ministry of Health, Labor, and Welfare (Japan).

Accepted for publication December 11, 2009.

Address reprint requests to Yasufumi Sato, M.D., Ph., Department of Vascular Biology, Institute of Development, Aging, and Cancer, Tohoku University, 4-1, Seiryo-machi, Aoba-ku, Sendai 980-8575, Japan. E-mail: y-sato@idac. tohoku.ac.jp. 
(IGF1), and hepatocyte growth factor (HGF) are reported to induce both angiogenesis and lymphangiogenesis. ${ }^{5-8}$

Angiogenesis is counterbalanced by various endogenous inhibitors. ${ }^{9}$ However, little is known about endogenous inhibitors of lymphangiogenesis. Thrombospondin 1 (TSP1), an angiogenesis inhibitor, does not inhibit lymphangiogenesis. ${ }^{10}$ Endostatin, another angiogenesis inhibitor, inhibits lymphangiogenesis and LN metastasis of certain tumors, but its effect on lymphangiogenesis is mediated via the down-regulation of VEGF-C in tumor cells. ${ }^{11,12}$

Recently, while searching for novel and functional VEGF-A-inducible molecules in endothelial cells (ECs), we identified an intrinsic inhibitor of angiogenesis in the vascular endothelium and named it vasohibin (VASH). ${ }^{13}$ Thereafter we isolated a homologue of VASH, and so we designated it as VASH2 and renamed the original VASH as VASH1. ${ }^{14}$ Our subsequent analysis revealed that VASH1 is expressed in BECs in the termination zone to halt angiogenesis, whereas VASH2 is expressed in infiltrating mononuclear cells in the sprouting front to promote angiogenesis. ${ }^{15}$ When applied exogenously, VASH1 effectively inhibits various kinds of pathological angiogenesis ${ }^{13,16-18}$ and inhibits tumor growth. ${ }^{13,18}$ Here, we examined whether VASH1 has any effect on lymphangiogenesis, and if so, on LN metastasis of tumors. Our present study provides evidence that intrinsic factor VASH1 exhibited broad-spectrum antilymphangiogenic activity and inhibited LN metastasis.

\section{Materials and Methods}

All of the animal studies were reviewed and approved by the committee for animal study at our institute in accord with established standards of humane handling of research animals.

\section{Mouse Corneal Micropocket Assay}

Mouse corneal micropocket assays were performed as described previously. ${ }^{13}$ Briefly, 4-week-old male BALB/c mice (Charles River Laboratories Japan, Inc., Yokohama, Japan) were deeply anesthetized, and $0.3 \mu \mathrm{g}$ of poly-2hydroxyethyl methacrylate (HEME) pellets (Sigma, St. Louis, Mo, USA) containing either vehicle or $160 \mathrm{ng}$ of VEGF-A (VEGF 165 , Sigma), 160 ng of VEGF-C Cys 156 ser (R\&D Systems, Inc., Minneapolis, MN), 12.5 ng or 80 ng of FGF2 (BD Biosciences, San Jose, CA), or $80 \mathrm{ng}$ of PDGF-BB (R\&D Systems, Inc.) was implanted in the corneas. A total of $4 \mathrm{ng}$ of VASH1 protein was added or not to the pellets.

Fourteen days after the pellet inoculation, the corneas were excised, washed in PBS, and fixed in acetone at $4^{\circ} \mathrm{C}$ for 30 minutes. After three additional washings in PBS and blocking with 1\% BSA in PBS for 1 hour, the corneas were stained overnight at $4^{\circ} \mathrm{C}$ with rabbit antimouse lymphatic vessel endothelial receptor 1 (LYVE1) antibody (1:500; Acris Antibodies GmbH, Hiddenhausen, Germany) and rat anti-mouse CD31 antibody (1:500; Research Diagnostics Inc., Flanders, NJ). On day 2, the corneas were washed, and secondary antibody reactions were performed by treatment with Alexa Fluor 488-conjugated donkey anti-rat IgG (1:1000; Invitrogen Corp., Carlsbad, CA) and Alexa Fluor 568-conjugated goat anti-rabbit IgG (1:1000; Invitrogen Corp.) for 6 hours at $4^{\circ} \mathrm{C}$. After a last washing, the sections were covered with fluorescence mounting medium (DakoCytomation Inc., Carpinteria, CA). Double-stained whole-mount sections were observed under a FluoView FV1000 confocal microscope (Olympus Corp., Tokyo, Japan). Blood vessels were positive for CD31 antigen, and lymphatic vessels were positive for LYVE1. The area covered by blood and lymphatic vessels was measured by using NIH ImageJ software (v. 1.39u).

\section{Subcutaneous Tumor Xenograft Model}

Cells of the human lung cancer cell line $\mathrm{NCl}-\mathrm{H} 460$ LNM35 (LNM35, $1.0 \times 10^{7}$ cells) were implanted into the subcutaneous tissue of the right abdominal wall of female SCID mice (6 to 8 weeks old, Charles River Laboratories, Japan). A replication-defective adenovirus vector encoding human vasohibin1 (AdVASH1) or $\beta$-galactosidase gene (AdLacZ, $1 \times 10^{9}$ plaque-forming units [pfu]) was intravenously injected into a tail vein at day 0 and day 14 after the implantation. ${ }^{17}$ Four weeks after the inoculation the mice were sacrificed, and tumors, along with some internal organs such as the trachea and axillary LNs, were collected. The sizes of axillary LNs were measured, and sections of the nodes were stained with hematoxylin and eosin to evaluate tumor metastasis.

Tissues were embedded in optimal cutting temperature (OCT) compound (Sakura Finetechnical, Tokyo, Japan) to make frozen tissue specimens, and sectioned at $6 \mu \mathrm{m}$. Samples were fixed with methanol for 20 minutes at $-20^{\circ} \mathrm{C}$, blocked with $1 \%$ BSA in PBS for 30 minutes at room temperature, and stained with anti-mouse LYVE-1 antibody (1:500), anti-mouse CD31 antibody (1:500), or anti-mouse F4/80 antibody (1:500; Acris Antibodies $\mathrm{GmbH})$ at $4^{\circ} \mathrm{C}$ overnight. This action was followed by staining with secondary antibodies Alexa fluor 488 donkey anti-rat lgG (1:1000), Alexa fluor 568 goat anti-rabbit IgG (1:1000) and TO-PRO-3 iodide (1:1000; Invitrogen Corp.) for 30 minutes at room temperature. After having been washed three times with PBS, the sections were covered with fluorescence mounting medium and observed under an Olympus FluoView FV1000 confocal microscope. The vascular lumen was traced, and the vascular luminal area was analyzed with $\mathrm{NIH}$ ImageJ software.

\section{Western Blotting of Human VASH1 Protein}

Frozen tissues (vena cava, kidney, liver, lung, and heart) were homogenized and lysed with modified RIPA buffer. Mouse blood was heparinized and centrifuged to obtain plasma. Albumin and IgG were depleted from the plasma with a removal kit according to the manufacturer's protocol (Amersham Biosciences Corp., Piscataway, NJ). Thereafter, Western blot analysis was performed as described previously. ${ }^{13}$ Horseradish peroxidase (HRP)-labeled anti-human VASH1 monoclonal antibody (clone $4 \mathrm{E} 12$ ) was used, which recognized human but not murine VASH1 protein. 


\section{ELISA for VASH1}

Peptides corresponding to Gly286-Arg299 (VC1) and Ala217-Lys229 (VR) of human VASH1 protein were conjugated with keyhole limpet hemocyanin. These antigens were used to immunize A/J mice, and several monoclonal antibodies were prepared as described previously. ${ }^{13} \mathrm{We}$ examined various combinations of monoclonal antibodies and found that the combination of VC1-derived clone 12F6 and VR-derived clone 12E7 was ideal for a highly sensitive and specific ELISA system that could detect human and mouse VASH1 protein equally. We used 12F6 and $12 \mathrm{E} 7$ for plate coating and HRP labeling, respectively. The detailed procedure for the measurement was described previously. ${ }^{19}$

\section{Preparation of Recombinant VASH1 Protein}

Human VASH1 gene with optimized codons for Eschericha coli (E. coli) expression was cloned in pET-32 $\mathrm{LIC/Xa}$ (Novagen, Madison, WI). The resultant expression plasmid encoded VASH1 with a sequence of GSNSPLAMAISDPNSSSVDKLAAALEHHHHHH at its $C$ terminus. $E$.

\section{A}
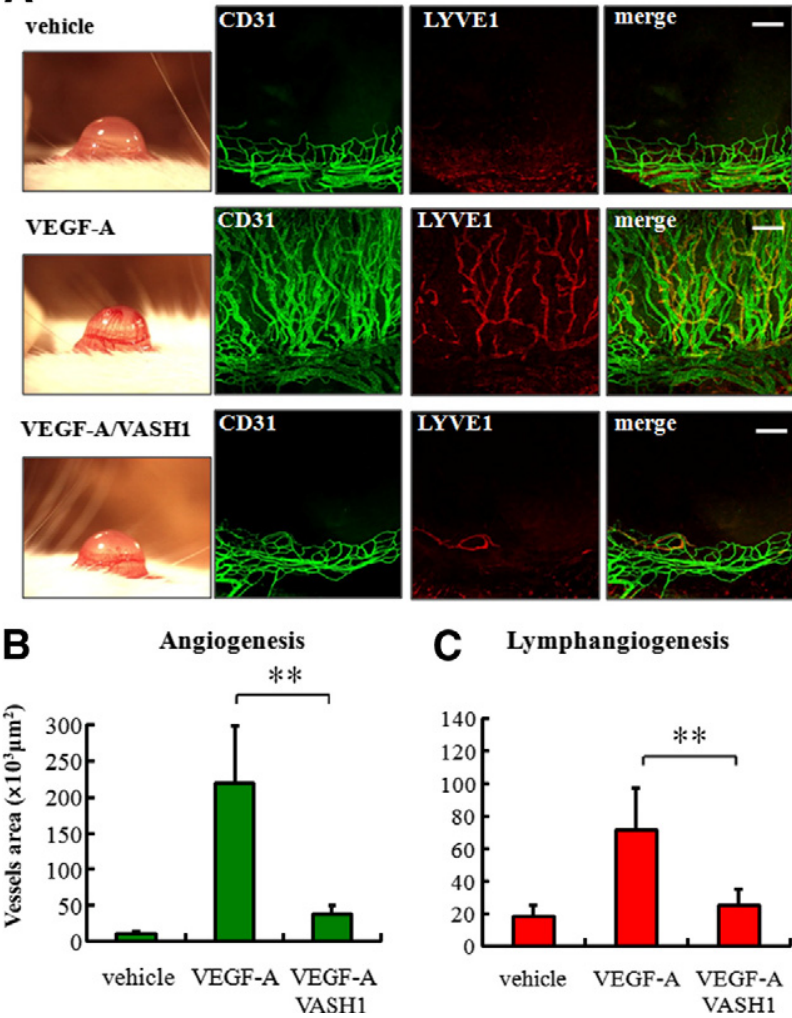

C Lymphangiogenesis

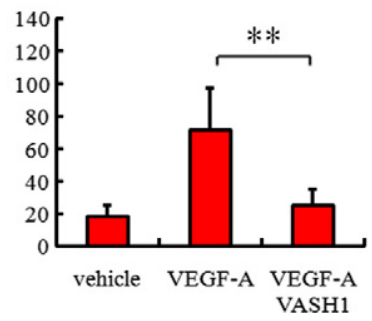

Figure 1. VASH1 inhibits angiogenesis and lymphangiogenesis induced by VEGF-A. A: Pellets containing 160 ng of VEGF-A plus or minus 4 ng of VASH1 were inoculated into the mouse cornea. Fourteen days after the inoculation, the corneas were harvested and immunostained for LYVE1 (red) or CD31 (green). Scale bar $=200 \mu \mathrm{m}$. Experiments were repeated at least 3 times, and representative data are shown here. B: The area of CD31-positive vessel was quantified; the means and SDs are shown. VASH1 significantly inhibited angiogenesis and lymphangiogenesis induced by VEGF-A. $n=5,{ }^{* * *} P<0.01$. C: The area occupied by LYVE1-positive vessel was quantified, and the means and SDs shown. VASH1 significantly inhibited the lymphangiogenesis induced by VEGF-A. $n=5,{ }^{* * *} P<0.01$. coli transformants were cultivated at $37^{\circ} \mathrm{C}$ in TB $(2.4 \mathrm{M}$ yeast extract, 1.2 Mtryptone, $1.25 \mathrm{M} \mathrm{K}_{2} \mathrm{HPO}_{4}, 0.23 \mathrm{M}$ $\mathrm{KH}_{2} \mathrm{PO}_{4}, 500 \mu \mathrm{g} / \mathrm{m} /$ polypropylene glycol \#2000, 50 $\mu \mathrm{g} / \mathrm{ml}$ ampicillin; $\mathrm{pH} 7.0$ ) supplied with $4 \%$ glycerol, and the expression was induced by the addition of $1 \mathrm{mmol} / \mathrm{L}$ isopropyl $\beta$-D-1-thiogalactopyranoside $\left(\mathrm{OD}_{650}=5\right)$. After a 16-hour cultivation, cells were collected and disrupted in $20 \mathrm{mmol} / \mathrm{L}$ sodium phosphate buffer, $\mathrm{pH} 7.6$, containing $0.5 \mathrm{~mol} / \mathrm{L} \mathrm{NaCl}$ and $1 \mathrm{mmol} / \mathrm{L}$ phenylmethylsulfonyl fluoride in a high-pressure homogenizer. The inclusion bodies were collected, washed with the same buffer, and solubilized in $20 \mathrm{mmol} / \mathrm{L}$ sodium phosphate buffer, $\mathrm{pH}$ 8.0 , containing $0.5 \mathrm{~mol} / \mathrm{L} \mathrm{NaCl}, 1 \mathrm{mmol} / \mathrm{L}$ phenylmethylsulfonyl fluoride, $5 \mathrm{mmol} / \mathrm{L}$ 2-mercaptoethanol, $60 \mathrm{mmol} / \mathrm{L}$ imidazole, and $7 \mathrm{~mol} / \mathrm{L}$ guanidine- $\mathrm{HCl}$. The soluble fraction was loaded onto a Ni Chelating Sepharose column (16 mm × $125 \mathrm{~mm}$, GE health care, Carnegie Center, NJ) equilibrated with the above solubilization buffer except that the guanidine- $\mathrm{HCl}$ was replaced by $8 \mathrm{~mol} / \mathrm{L}$ urea and eluted with the same buffer containing $300 \mathrm{mmol} / \mathrm{L}$ imidazole. VASH1 fusion protein fraction was dialyzed against $20 \mathrm{mmol} / \mathrm{L}$ glycine- $\mathrm{HCl}$ buffer, $\mathrm{pH} \mathrm{3.5,} \mathrm{and} \mathrm{di-}$ gested with coagulation factor Xa (Novagen) for 1 hour at
A
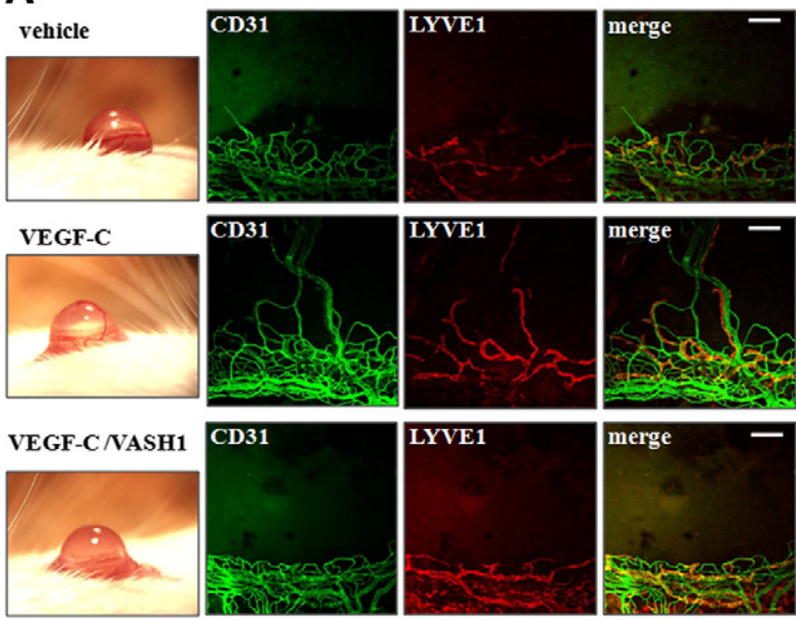

B

Angiogenesis
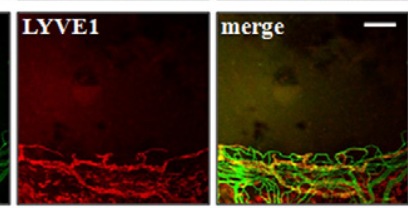

C Lymphangiogenesis
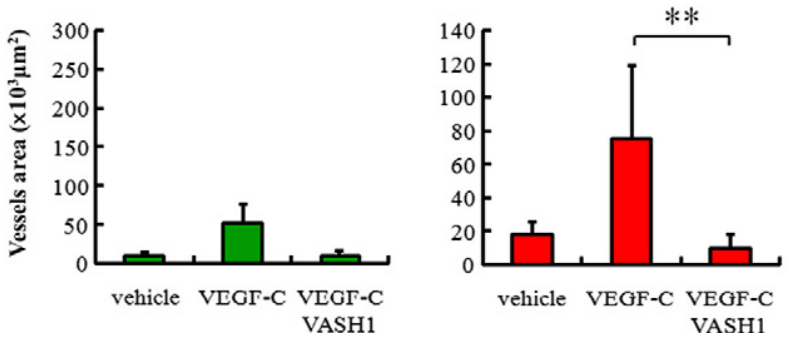

Figure 2. VASH1 inhibits angiogenesis and lymphangiogenesis induced by VEGF-C. A: Pellets containing 160 ng of VEGF-C plus or minus 4 ng of VASH1 were inoculated into the mouse cornea. Fourteen days after the inoculation, corneas were harvested and immunostained for LYVE1 or CD31. Scale bar = $200 \mu \mathrm{m}$. Experiments were repeated at least 3 times, and representative data are shown here. B: The area of CD31-positive vessel was quantified, and the means and SDs are shown. VEGF-C limitedly stimulated angiogenesis, as did VASH1, though no significant differences were observed. $n=5$. C: The area of LYVE1-positive vessels was quantified, and the means and SDs are shown. VASH1 significantly inhibited the lymphangiogenesis induced by VEGF-C. $n=5,{ }^{* * *} P<0.01$. 
$25^{\circ} \mathrm{C}$. The released $\mathrm{VASH} 1$ protein was collected, solubilized, and purified with Ni Chelating Sepharose. VASH1 protein was then collected as the insoluble fraction after dialysis against $20 \mathrm{mmol} / \mathrm{L}$ Tris- $\mathrm{HCl}(\mathrm{pH} \mathrm{8.0)}$, resolubilized in $25 \mathrm{mmol} / \mathrm{L}$ sodium phosphate $(\mathrm{pH} 7.2)$ containing $4 \mathrm{~mol} / \mathrm{L}$ urea, loaded onto a $\mathrm{Q}$ Sepharose column (16 $\mathrm{mm} \times 140 \mathrm{~mm}$, GE health care), and eluted by linearly increasing the $\mathrm{NaCl}$ concentration to $1 \mathrm{~mol} / \mathrm{L}$. Finally, the VASH1 protein was dialyzed against $20 \mathrm{mmol} / \mathrm{L}$ glycine$\mathrm{HCl}$ buffer ( $\mathrm{pH}$ 3.5).

\section{Orthotopic Tumor Xenograft Model}

Human breast cancer cell line MDA-MB-231 obtained from American Type Culture Collection was transfected with firefly luciferase and geneticin resistance genes, and stable transfectants (231Luc-1 cells) were selected. 231 Luc-1 cells were inoculated into the mammary fat pad of mice, and spontaneous LN metastatic cells (231LN-Luc-1 cells) were isolated from the axillary $L N s$ and cultured.

231 LN-Luc- 1 cells $\left(5 \times 10^{6}\right)$ in $50 \mu \mathrm{l}$ of $40 \%$ Hanks' balanced salt solution containing 50\% Matrigel (Becton,
Dickinson and Company, Franklin Lakes, NJ) and 10\% VASH1 or human serum albumin (HSA) solution ( $5 \mu \mathrm{g}$ protein/50 $\mu$ l solution) were inoculated into the abdominal mammary fat pad of female C57BL-17/Icr SCID JCl mice (CLEA Japan, Inc., Tokyo, Japan). Six days after the inoculation, $2.5 \mu \mathrm{g}$ of VASH1 or HSA was locally injected into the abdominal mammary fat pad every 3 to 4 days. LN metastasis (axillary region) was analyzed on day 32 by a bioluminescence imaging technique. Fifty to 60 seconds after the luciferin injection, mice were placed in the IVIS Imaging System (Xenogen, Alameda, CA) and imaged. LN metastasis was quantified as photons/sec obtained with Living Image ${ }^{\circledR}$ software (Xenogen).

\section{Calculations and Statistical Analysis}

Data were expressed as the mean plus or minus SD. The significance of the data were determined by using Student $t$ test for the evaluation of angiogenesis, lymphangiogenesis, plasma VASH1 concentration, and tumor-related photons, and by performing Fisher exact test
A
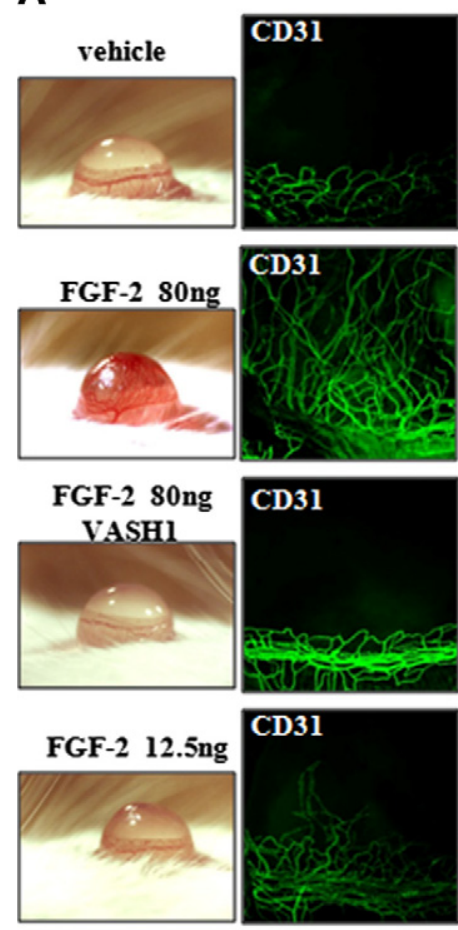

FGF-2 12.5 ng VASHl
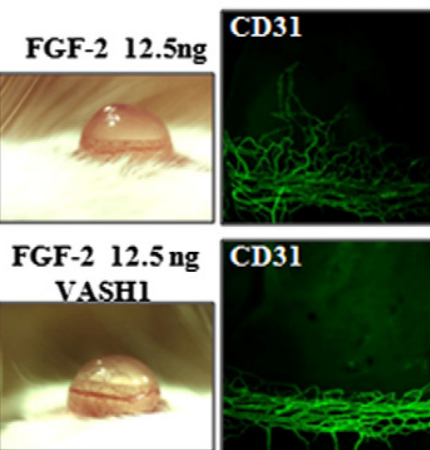
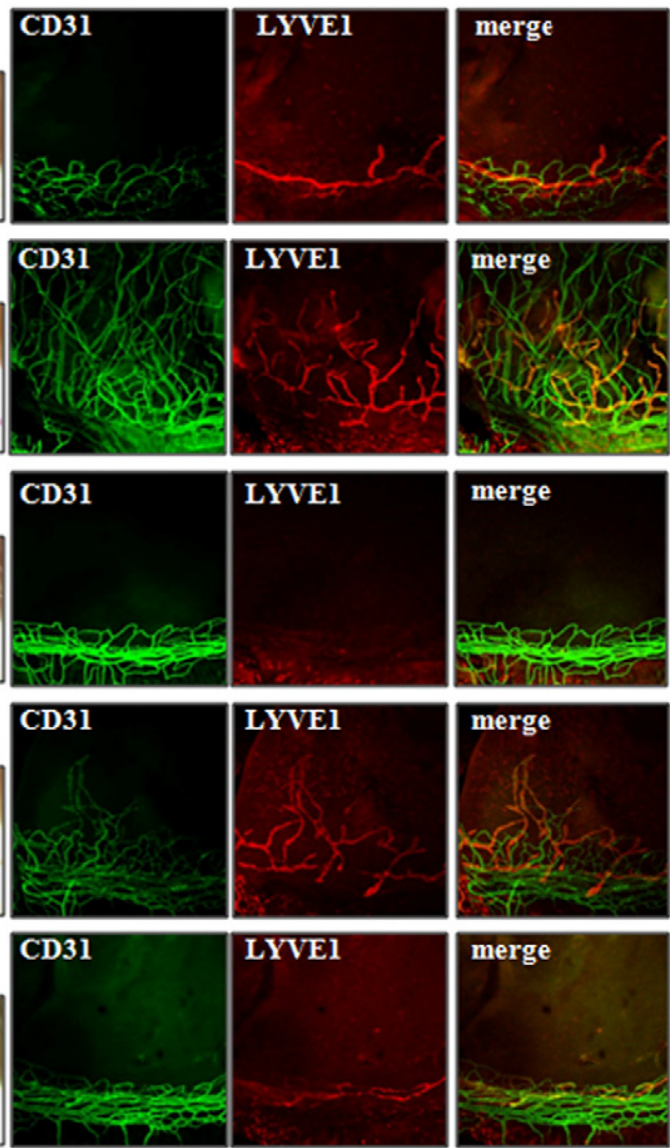

\section{LYVE1}
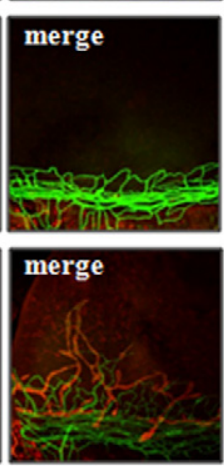

merge

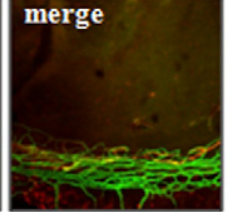

B
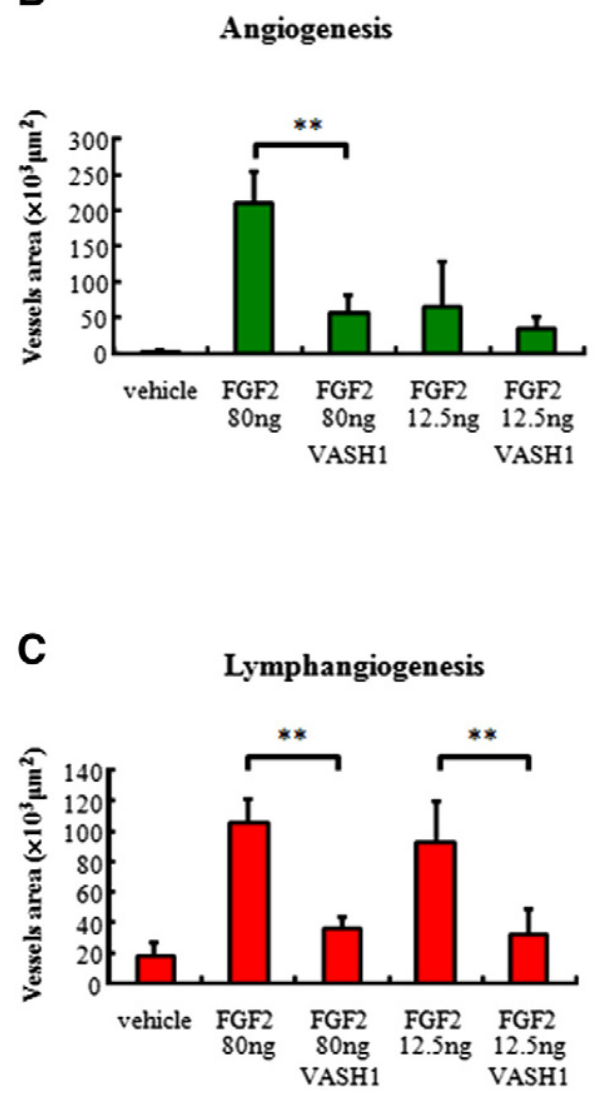

Figure 3. VASH1 inhibits angiogenesis and lymphangiogenesis induced by FGF2. A: Pellets containing 80 ng (high dose) or 12.5 ng (low dose) of FGF2 plus or minus 4 ng of VASH1 were inoculated into the mouse cornea. Fourteen days after the inoculation, the corneas were harvested and immunostained for LYVE1 (red) or CD31 (green). Scale bar $=200 \mu \mathrm{m}$. Experiments were repeated at least 3 times, and representative data are shown here. B: The areas of CD31-positive vessel were quantified, and the means and SDs are shown. At the higher dose, FGF2 induced angiogenesis, and VASH1 significantly inhibited the angiogenesis induced by FGF2. $n=5,{ }^{* *} P<0.01$. At the lower dose, FGF2 did not significantly induce angiogenesis. $n=5$. C: The areas of LYVE1-positive vessel were quantified, and the means and SDs are shown. At both the higher and lower doses, FGF2 induced lymphangiogenesis, and VASH1 significantly inhibited the lymphangiogenesis induced by either dose of FGF2. $n=5,{ }^{*} P<<0.01$. 
for the evaluation of lymph node metastasis. Statistical significance was defined as a $P$ value less than 0.05 .

\section{Results}

VASH1 Exhibits Broad-Spectrum Antiangiogenic and Antilymphangiogenic Activities

Earlier we used human VASH1 protein in various mouse models and showed its antiangiogenic activity. ${ }^{13,16-18}$ A recent study indicated that the antiangiogenic effect of mouse VASH1 protein was not distinguishable from that of human VASH1 protein in a mouse model. ${ }^{20}$ Here we used human VASH1 protein. VEGF-A strongly induces both angiogenesis and lymphangiogenesis in the mouse cornea. ${ }^{21}$ By immunostaining a mouse cornea for CD31 as a marker for BECs and for LYVE1 as a marker for LECs, we confirmed this activity of VEGF-A (Figure 1A), and further showed that the co-administration of recombinant VASH1 protein with VEGF-A almost completely blocked VEGF-A-induced angiogenesis and lymphangiogenesis (Figure 1, B and C).

We next applied VEGF-C, a principal stimulator of lymphangiogenesis, to the mouse cornea. In agreement with a previous report, ${ }^{5}$ VEGF-C induced lymphangiogenesis and also angiogenesis to some extent when administered alone to mouse corneas, and co-administration of VASH1 with VEGF-C abolished both lymphangiogenesis and angiogenesis induced by VEGF-C (Figure 2A). Quantitative analysis confirmed these effects of VASH1 (Figure 2, B and C).

We further administered growth factors other than VEGF family members that are known to have stimulatory effects on angiogenesis and lymphangiogenesis. It is reported that FGF2 induces both angiogenesis and lymphangiogenesis at a higher dose (80 ng per pellet), but primarily induces lymphangiogenesis at a lower dose (12.5 ng per pellet). ${ }^{22}$ We confirmed these differential effects of FGF-2 and further demonstrated that co-administration of VASH1 with high-dose FGF2 almost completely blocked both angiogenesis and lymphangiogenesis (Figure 3, A-C). PDGF-BB is reported to induce intratumoral lymphangiogenesis and to promote lymphatic metastasis. ${ }^{6}$ VASH1 inhibited both angiogenesis and lymphangiogenesis induced by PDGF-BB (Figure 4, $A$ and $B$ ).

Taken together, these results indicate that VASH1 has broad-spectrum antiangiogenic and antilymphangiogenic activities.

\section{VASH1 Inhibits Tumor Lymphangiogenesis and LN Metastasis}

Next, we proceeded to test the effect of VASH1 in the tumor xenograft model. We injected adenovirus vector encoding the human VASH1 gene (AdVASH1) into a tail vein of mice. Adenovirus vector encoding the $\beta$-galac-
A

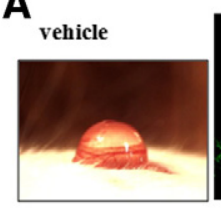

CD31
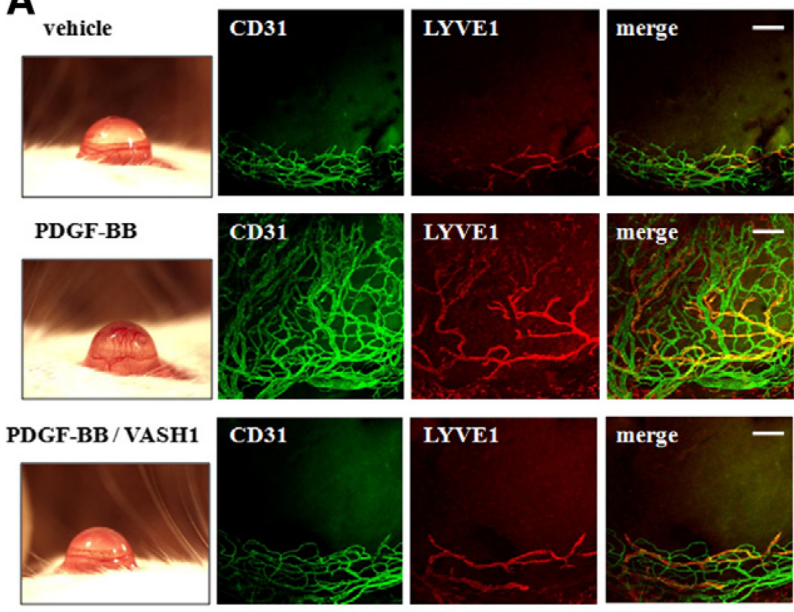

B

Angiogenesis
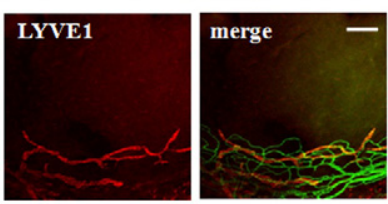

Lymphangiogenesis
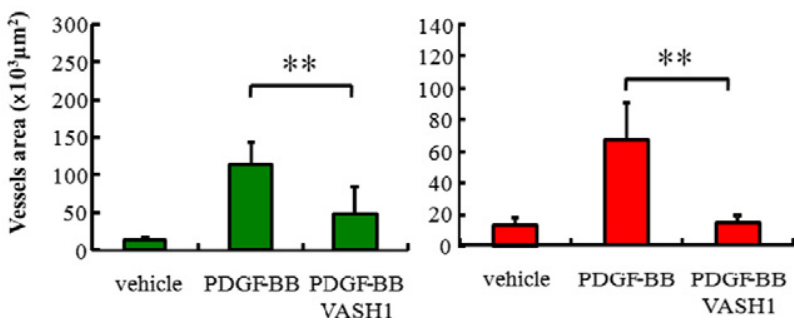

Figure 4. VASH1 inhibits angiogenesis and lymphangiogenesis induced by PDGF-BB. A: Pellets containing $160 \mathrm{ng}$ of PDGF-BB plus or minus $4 \mathrm{ng}$ of VASH1 were inoculated into the mouse cornea. Fourteen days after the inoculation, corneas were harvested and immunostained for LYVE1 or CD31. Scale bar $=200 \mu \mathrm{m}$. Experiments were repeated at least three times, and representative data are shown here. B: The area of CD31positive vessel was quantified, and the means and SDs are shown. VASH1 significantly inhibited the angiogenesis induced by PDGF-BB. $n=5$, ${ }^{* * *} P<0.01$. The area of LYVE1-positive vessel was quantified, with means and SDs shown. VASH1 significantly inhibited the lymphangiogenesis induced by PDGF-BB. $n=5, * * P<0.01$.

tosidase gene (AdLacZ) was used as a negative control. This vector should supply sufficient VASH1 protein to regulate angiogenesis, as described previously. ${ }^{17}$ Indeed, Western blotting for human VASH1 revealed that human VASH1 protein accumulated in various organs 10 days after the viral injection (Figure 5A). Differences in molecular size should be attributable to the FLAG tag in recombinant VASH1 protein for control, ${ }^{11}$ and posttranslational processing of $\mathrm{VASH} 1$ protein in mice. ${ }^{19}$ ELISA analysis recognizing both murine and human $\mathrm{VASH} 1$ revealed that the plasma concentration of VASH1 increased about threefold (Figure 5B).

We then inoculated the flanks of SCID mice with highly LN-metastatic human non-small cell lung cancer (LNM35) cells. $^{23}$ Adenovirus vectors were injected on day 0 and day 14, and tumor tissues were collected on day 28. Tumor angiogenesis was analyzed by immunostaining VECs for CD31. Blood vessels were distributed within the tumor; and, as expected, the blood vessel area was significantly reduced in the AdVASH1-injected group (Figure 5C). Tumor lymphangiogenesis was analyzed by immunostaining for LYVE1. We simultaneously performed F4/80 immunostaining to distinguish LYVE1-expressing macrophages as described. ${ }^{24}$ LYVE1-positive and F4/ 
A

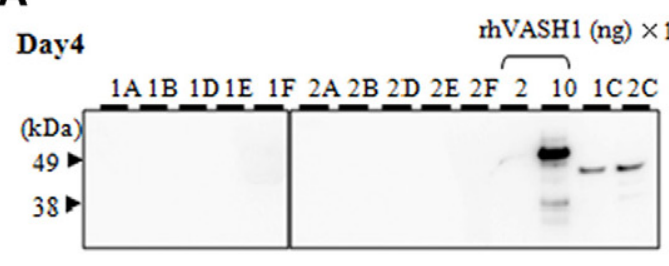

Daylo

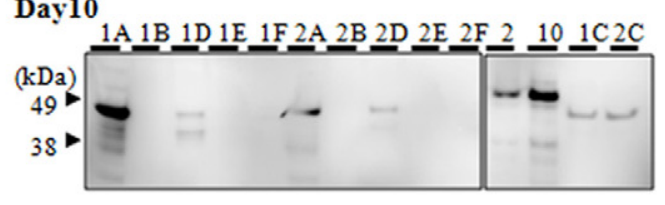

C

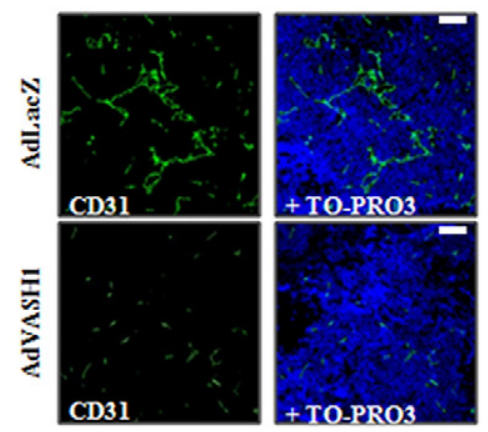

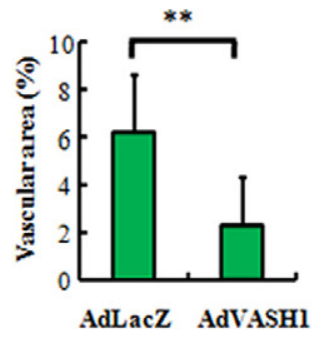

B

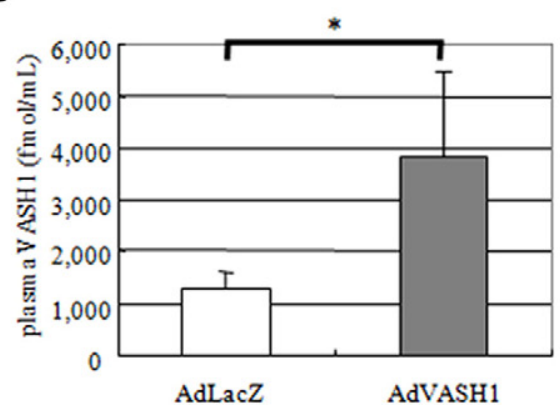

D

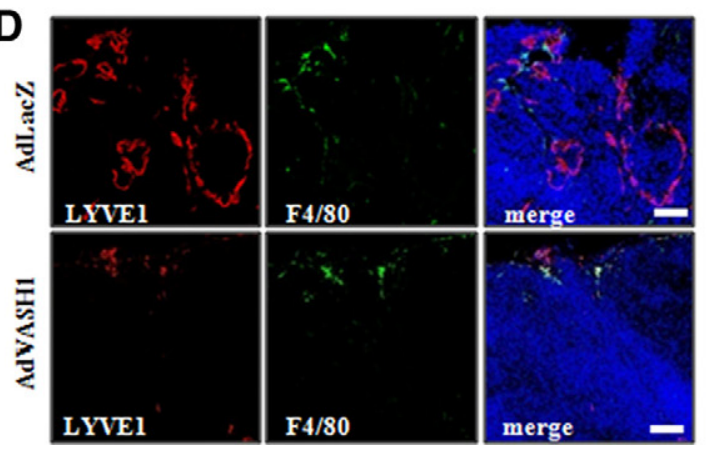

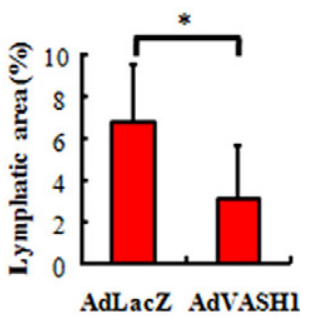

Figure 5. Adenovirus-mediated systemic delivery of VASH1 protein inhibits tumor lymphangiogenesis of LNM 35 cells. A: AdVASH 1 or AdLacZ ( $1 \times 10^{9}$ pfu) was injected into a tail vein of mice. Various organs were collected from two mice on day 4 and day 10 after the injection, and Western blotting for human VASH1 protein was performed as described in Materials and Methods. Recombinant human VASH1 protein with a triple repeat of the FLAG tag (rhVASH1) was used as a control. B: Plasma samples were collected 10 days after the injection, and the concentration of VASH1 was determined by ELISA. $n=5$, ${ }^{*} P<0.05$. C: LNM 35 cells were inoculated subcutaneously, and adenovirus vectors were injected into the tail vein of mice on day 0 and day 14 . On day 28 , the mice were sacrificed and tumors were resected. Tumor sections were immunostained for CD31 (green), and TO-PRO3 (blue) was used for nuclear staining. Scale bar $=200 \mu \mathrm{m}$. The intratumoral CD31-positive vascular area was quantified and expressed as \% of a field. The means and SDs are shown. Tumor angiogenesis was significantly inhibited in the AdVASH1-injected mice. $n=13$ (AdLacZ), $n=12$ (AdVASH1), ${ }^{* *} P<0.01$. D: Tumor sections were immunostained for LYVE1 (red) and F4/80 (green). TO-PRO3 (blue) was used for nuclear staining. Scale bar $=100 \mu \mathrm{m}$. The peritumoral LYVE1-positive and F4/80-negative vascular area was quantified and expressed as $\%$ of a field. The means and SDs are shown. Tumor lymphangiogenesis was significantly inhibited in the AdVASH1-injected mice, $n=13$ (AdLacZ), $n=12$ (AdVASH1), ${ }^{*} P<0.05$.

80-negative lymphatic vessels were distributed in the peri-tumoral region (Figure 5D). Quantitative analysis revealed that lymphatic vessels in the peritumoral region were significantly reduced in area in the AdVASH1-injected group (Figure 5D).

The regional axillary LNs were recovered on day 28 . LN size was measured, and LN metastasis was determined by histological analysis. VASH1 significantly inhibited LN metastasis, as LN metastasis occurred in 14 of 17 AdLacZ-injected mice, but in only 4 of 16 AdVASH1injected mice (Figure 6A). It has been described that lymphangiogenesis in the regional LNs occurs before LN metastasis and determines tumor dissemination beyond the regional $L N s .{ }^{25,26}$ Recovered $L N s$ were therefore immunostained for CD31 and LYVE1 to analyze angiogenesis and lymphangiogenesis (Figure 6B). Angiogenesis was not increased in either metastasis-negative or metastasis-positive LNs when compared with LNs isolated from non-tumor-bearing mice (normal LNs), but was significantly decreased in AdVASH1injected mice when compared with the AdLacZ-injected mice (Figure 6C). In contrast, lymphangiogenesis was sig- nificantly augmented in the metastasis-negative LNs of the AdLacZ-injected mice, and was abolished in those the AdVASH1-injected mice (Figure 6C). These results suggest that VASH1 inhibited lymphangiogenesis in regional LNs before the establishment of LN metastasis.

We tested whether VASH1 impaired normal vessels in mice. Tracheal mucosa was immunostained for CD31 and LYVE1. We did not detect any morphological changes in blood or lymphatic vessels of mice injected with AdVASH1 (Figure 7A) Quantitative analysis revealed that VASH1 did not alter the area of blood or lymphatic vessels (Figure 7B).

To further show the effect of VASH1 on LN metastasis, we performed orthotopical inoculation of LN metastatic human breast cancer (231LN-Luc-1) cells. Moreover, because of the limitation of the gene therapy approach in cancers, we applied recombinant VASH1 protein. We inoculated 231LN-Luc-1 cells into the abdominal mammary fat pad of mice in the presence of recombinant VASH1 protein. We then injected recombinant VASH1 protein locally, because of the obstacle of using recombinant VASH1 protein for systemic administration. There 
A

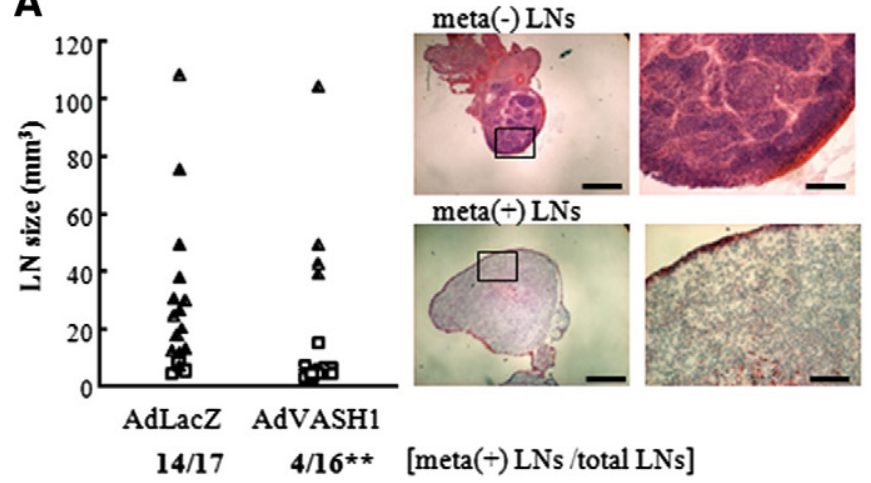

C
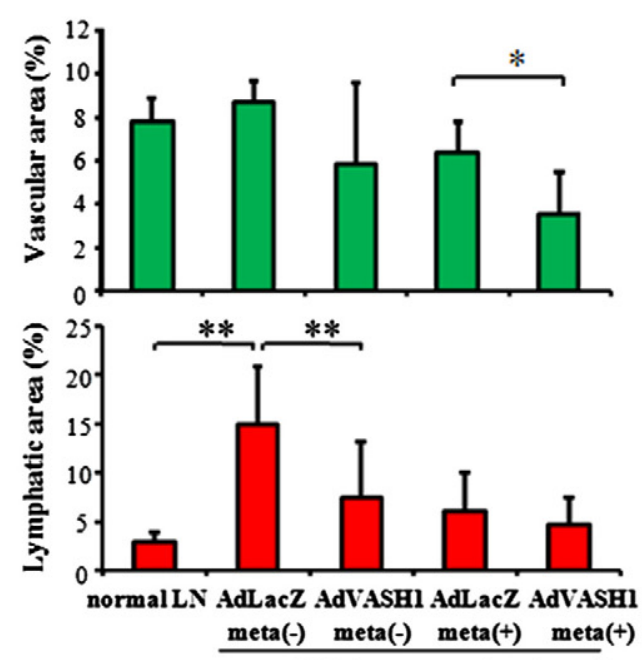

B
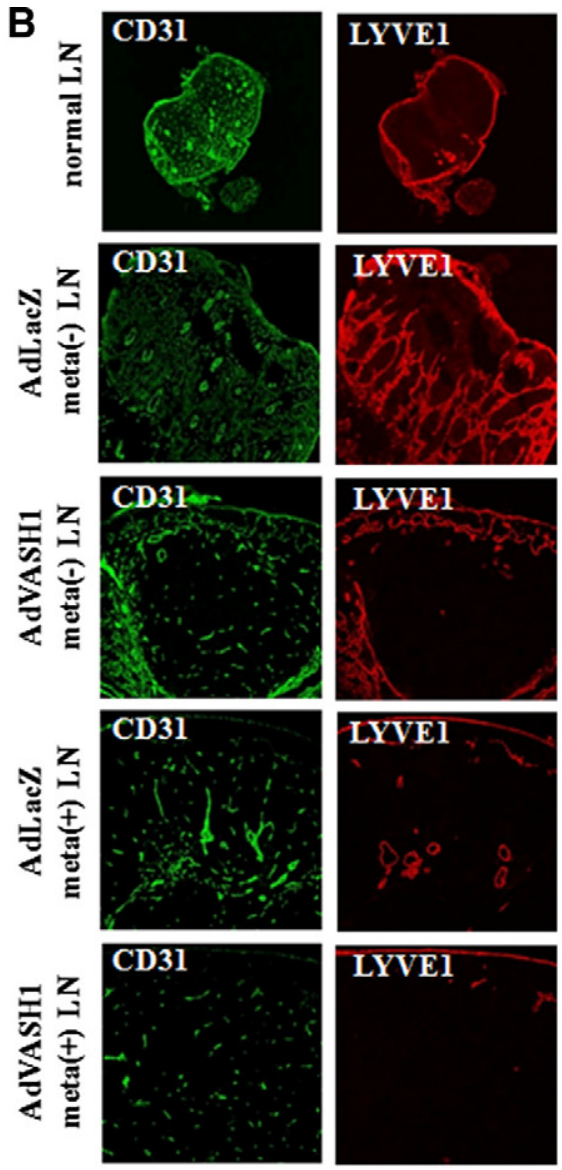
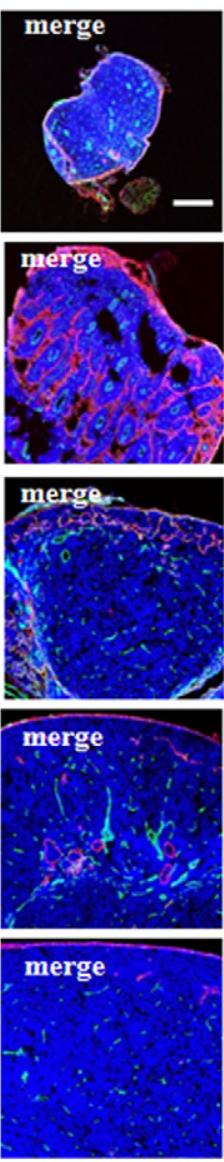

LNs from tumor-bearing mice

Figure 6. VASH1 inhibits LN metastasis of subcutaneously inoculated LNM35 cells. A: Axillary LNs were recovered. LN size was measured, and the size of LNs and the presence or absence of LN metastasis is shown. The frequency of LN metastasis in AdVASH1-injected mice was significantly lower, ** $P<0.01$. Experiments were repeated three times, and representative data are shown here. LN metastasis was determined by histological analysis. Boxed fields on the left were enlarged and shown on the right. Scale bars $=500 \mu \mathrm{m}$ on the left and $100 \mu \mathrm{m}$ on the right. B: LNs were immunostained for CD31 (green) and LYVE1 (red). TO-PRO3 (blue) was used for nuclear staining. Scale bar $=200 \mu \mathrm{m}$. C: The CD31-positive and LYVE1-positive vessel areas were quantified and expressed as \% of a field. The means and SDs are shown. The area of lymphatic vessels was significantly decreased in the metastasis-negative LNs of the AdVASH1-injected mice, whereas the area of blood vessels was significantly decreased in the metastasis-positive LNs of the AdVASH1-injected mice. $n=8$ (AdLacZ meta + and AdVASH1 meta-), $n=4$ (normal LN, AdLacZ meta- and AdVASH1 meta+), ${ }^{*} P<0.05,{ }^{* * *} P<0.01$.

was a significant decreased in the bioluminescence in the VASH1-injected group (Figure 8).

\section{Discussion}

VASH1 was originally isolated as a VEGF-A-inducible angiogenesis inhibitor. ${ }^{13}$ Here we assessed the effect of VASH1 on lymphangiogenesis, and explored its broad-spectrum antiangiogenic and antilymphangiogenic activities. Our findings are the first demonstration that a molecule intrinsic to the endothelium exhibits such activities.

We first applied VASH1 in combination with VEGF-A to the mouse cornea. VEGF-A induced both angiogenesis and lymphangiogenesis, and co-administration of VASH1 abolished those effects of VEGF-A. VEGF-A can induce lymphangiogenesis by affecting LECs directly or indirectly. ${ }^{27,28}$ One means by which VEGF-A indirectly induces lymphangiogenesis is by mediating angiogenesis, which increases the local accumulation of inflammatory cells and thus the supply of lymphangiogenic factors such as VEGF-C. ${ }^{29-31}$ Because VASH1 inhibited angiogenesis, VASH1 might exhibit its antilymphangiogenesis activity via the indirect route. To further clarify the effect of VASH1 on lymphangiogenesis, we replaced VEGF-A with VEGF-C, a principal and direct lymphangiogenesis stimulator. The result showing that VASH1 inhibited VEGF-Cstimulated lymphangiogenesis supports the direct antilymphangiogenesis activity of VASH1. Notably, VASH1 inhibited FGF2- and PDGF-BB-induced angiogenesis and lymphangiogenesis as well. Thus, the inhibitory effect of VASH1 is not restricted to the phenomena induced by the VEGF family members.

We focused our attention on the antilymphangiogenic activity of VASH1, as tumor lymphangiogenesis is recognized as a therapeutic target for the prevention of LN metastasis. We experimentally used an adenovirus vector, and showed that VASH1 delivered by this means inhibited tumor lymphangiogenesis and LN metastasis in a mouse xenograft model. The injection of 
A
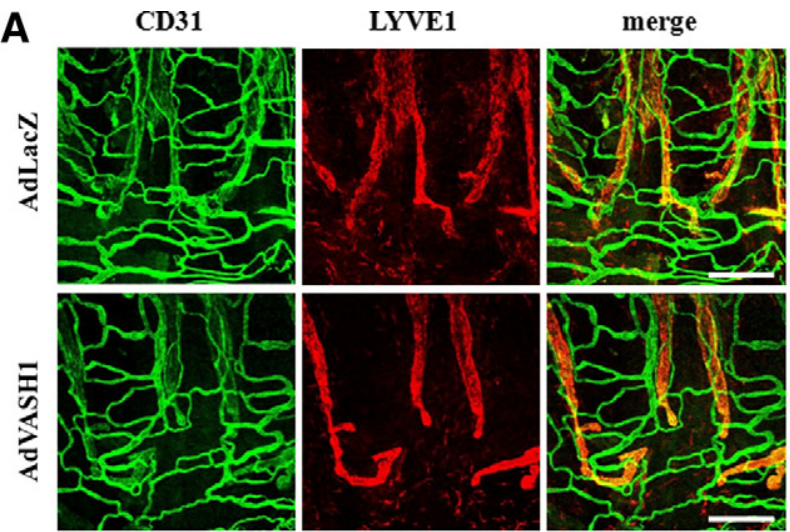

B

Blood Vessels

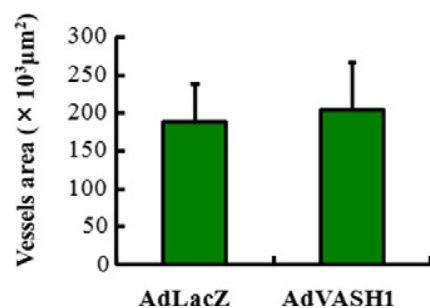

AdLacZ AdVASH1

Figure 7. VASH1 does not damage normal vessels. A: A mouse trachea was collected on day 28, and its mucosa was immunostained for CD31 (green) and LYVE1 (red). Scale bar $=200 \mu \mathrm{m}$. No morphological changes were evident in the AdVASH1-injected mice. B: The areas of blood vessels and lymphatic vessels were quantified, and the means and SDs are shown. $n=5$. No changes in blood or lymphatic vascular areas were evident.

AdVASH1 via a tail vein caused the synthesis of VASH1 protein in the liver. The accumulation of human VASH1 protein detected in various organs indicates that this procedure allowed us to supply human VASH1 protein systemically. The high affinity of $\mathrm{VASH} 1$ for heparin should be the reason for this local accumulation. ${ }^{19}$

As expected, AdVASH1 inhibited tumor lymphangiogenesis and regional LN metastasis. We further tested lymphatic vessels in the regional LNs, and found that lymphangiogenesis was significantly augmented in the metastasis-negative LNs and was inhibited by AdVASH1. However, lymphangiogenesis was no more augmented in the metastasis-positive LNs in the AdLacZ control. We speculate that this decrease in lymphatic vessels in the metastasis-positive LNs in the AdLacZ control might be attributable to the occupation of LNs by metastatic cancer tissues (Figure 6A), as lymphatic vessels are rarely present within cancer tissues. ${ }^{32}$

We previously reported the antitumor effect of VASH1 to occur through inhibition of angiogenesis. ${ }^{13,18}$ Antiangiogenic therapy is currently being used clinically to inhibit tumor angiogenesis and tumor growth by targeting VEGF-A-mediated signaling, but one of the problems with this treatment is drug resistance. ${ }^{33,34}$ Cancer cells switch to producing other factors such as FGF2 to combat the antiangiogenic therapy when they are treated with VEGF-A targeting monotherapy. ${ }^{33}$ PDGF-mediated signaling is another pathway that is activated in cancers. ${ }^{35}$ Importantly, FGF2 and PDGF-BB synergistically promote tumor neovascularization and distant metastasis. ${ }^{36}$ The fact that VASH1 exhibited broad-spectrum antiangiogenic activity, including that against FGF2 and PDGF-BB, reveals an advantageous characteristic of it.

It has been reported that the blockade of VEGFR3 signaling inhibits tumor lymphangiogenesis and LN metastasis. ${ }^{37}$ Thus, with analogy to the antiangiogenesis therapy, VEGFR3 signaling is proposed to be an appropriate target for the inhibition of lymphangiogenesis. It is not clear yet whether resistance would occur when VEGFR3 signaling is blocked. ${ }^{37}$ Nevertheless, because FGF2 and PDGF-BB, which are candidates to cause drug resistance in antiangiogenic therapy, promote lymphangiogenesis as well, the broad-spectrum antiangiogenic and antilymphangiogenic activities of VASH1 are noteworthy.

In summary, our present study shows that the intrinsic factor VASH1 has broad-spectrum antiangiogenic and antilymphangiogenic activities, thus affording it the potential to inhibit tumor lymphangiogenesis and LN metastasis. We propose that VASH1 should be tested further for controlling tumor angiogenesis and lymphangiogenesis.

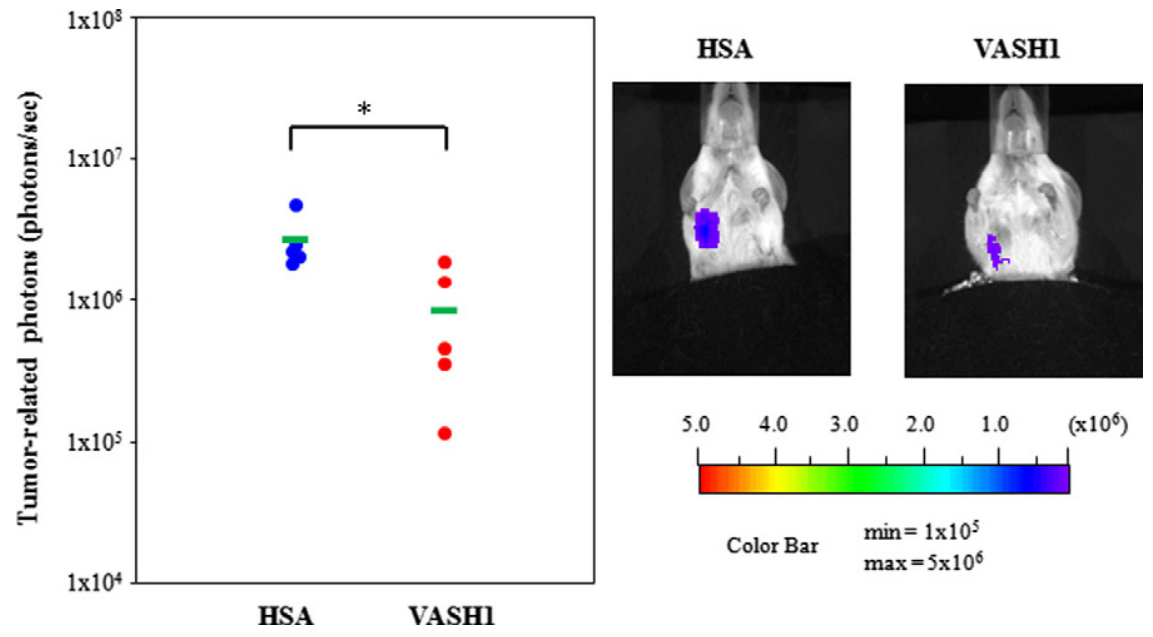

Figure 8. VASH1 inhibits LN metastasis of orthotopically inoculated 231LN-Luc-1 cells. 231LNLuc-1 cells $\left(5 \times 10^{6}\right)$ were inoculated into the abdominal mammary fat pad of mice. Recombinant VASH1 protein or HSA was applied locally. Auxiliary LN metastasis was analyzed by the bioluminescence imaging technique. LN metastasis on day 32 was quantified as photons/sec on the left. $n=5,{ }^{*} P<0.05$. Representative photos on day 32 are shown on the right. The color-bar indicates the value (photons/sec) for metastatic tumors. 


\section{Acknowledgments}

We thank Yuriko Fujinoya and Kyoko Shimizu for their excellent technical assistance.

\section{References}

1. Oliver G, Alitalo K: The lymphatic vasculature: recent progress and paradigms. Annu Rev Cell Dev Biol 2005, 21:457-483

2. Alitalo K, Tammela T, Petrova TV: Lymphangiogenesis in development and human disease. Nature 2005, 438:946-953

3. Leong SP, Cady B, Jablons DM, Garcia-Aguilar J, Reintgen D, Jakub J, Pendas S, Duhaime L, Cassell R, Gardner M, Giuliano R, Archie V, Calvin D, Mensha L, Shivers S, Cox C, Werner JA, Kitagawa Y, Kitajima M: Clinical patterns of metastasis. Cancer Metastasis Rev 2006, 25:221-232

4. Adams $\mathrm{RH}$, Alitalo $\mathrm{K}$ : Molecular regulation of angiogenesis and lymphangiogenesis. Nat Rev Mol Cell Biol 2007, 8:464-478

5. Cao R, Eriksson A, Kubo H, Alitalo K, Cao Y, Thyberg J: Comparative evaluation of FGF-2-. VEGF-A-, and VEGF-C-induced angiogenesis, lymphangiogenesis, vascular fenestrations, and permeability. Circ Res 2004, 94:664-670

6. Cao R, Bjorndahl MA, Religa P, Clasper S, Garvin S, Galter D, Meister B, Ikomi F, Tritsaris K, Dissing S, Ohhashi T, Jackson DG, Cao Y: PDGF-BB induces intratumoral lymphangiogenesis and promotes lymphatic metastasis. Cancer Cell 2004, 6:333-345

7. Bjorndahl M, Cao R, Nissen LJ, Clasper S, Johnson LA, Xue Y, Zhou $Z$, Jackson D, Hansen AJ, Cao Y: Insulin-like growth factors 1 and 2 induce lymphangiogenesis in vivo. Proc Natl Acad Sci USA 2005 102:15593-15598

8. Cao R, Bjorndahl MA, Gallego MI, Chen S, Religa P, Hansen AJ, Cao $Y$ : Hepatocyte growth factor is a lymphangiogenic factor with an indirect mechanism of action. Blood 2006, 107:3531-3536

9. Sato Y: Update on endogenous inhibitors of angiogenesis. Endothelium 2006, 13:147-155

10. Hawighorst $T$, Oura $H$, Streit M, Janes L, Nguyen L, Brown LF, Oliver G, Jackson DG, Detmar M: Thrombospondin-1 selectively inhibits early-stage carcinogenesis and angiogenesis but not tumor lymphangiogenesis and lymphatic metastasis in transgenic mice. Oncogene 2002, 21:7945-7956

11. Fukumoto S, Morifuji M, Katakura Y, Ohishi M, Nakamura S: Endostatin inhibits lymph node metastasis by a down-regulation of the vascular endothelial growth factor $C$ expression in tumor cells. Clin Exp Metastasis 2005, 22:31-38

12. Brideau G, Makinen MJ, Elamaa H, Tu H, Nilsson G, Alitalo K, Pihlajaniemi T, Heljasvaara R: Endostatin overexpression inhibits lymphangiogenesis and lymph node metastasis in mice. Cancer Res 2007, 67:11528-11535

13. Watanabe K, Hasegawa Y, Yamashita H, Shimizu K, Ding Y, Abe M, Ohta H, Imagawa K, Hojo K, Maki H, Sonoda H, Sato Y: Vasohibin as an endothelium-derived negative feedback regulator of angiogenesis. J Clin Invest 2004, 114:898-907

14. Shibuya T, Watanabe K, Yamashita H, Shimizu K, Miyashita H, Abe M, Moriya T, Ohta H, Sonoda H, Shimosegawa T, Tabayashi K, Sato Y: Isolation and characterization of vasohibin-2 as a homologue of VEGF-inducible endothelium-derived angiogenesis inhibitor vasohibin. Arterioscler Thromb Vasc Biol 2006, 26:1051-1057

15. Kimura H, Miyashita H, Suzuki Y, Kobayashi M, Watanabe K, Sonoda $\mathrm{H}$, Ohta H, Fujiwara T, Shimosegawa T, Sato Y: Distinctive localization and opposed roles of vasohibin-1 and vasohibin-2 in the regulation of angiogenesis. Blood 2009, 113:4810-4818

16. Shen JK, Yang XR, Sato Y, Campochiaro PA: Vasohibin is up-regulated by VEGF in the retina and suppresses VEGF receptor 2 and retinal neovascularization. FASEB J 2006, 20:723-725

17. Yamashita H, Abe M, Watanabe K, Shimizu K, Moriya T, Sato A, Satomi S, Ohta H, Sonoda H, Sato Y: Vasohibin prevents arterial neointimal formation through angiogenesis inhibition. Biochem Biophys Res Commun 2006, 345:919-925

18. Hosaka T, Kimura H, Heishi T, Suzuki Y, Miyashita H, Ohta H, Sonoda $H$, Moriya T, Suzuki S, Kondo T, Sato Y: Vasohibin-1 expressed in endothelium of tumor vessels regulates angiogenesis. Am J Pathol 2009, 175:430-439
19. Sonoda $H$, Ohta $H$, Watanabe K, Yamashita H, Kimura H, Sato $Y$ : Multiple processing forms and their biological activities of a novel angiogenesis inhibitor vasohibin. Biochem Biophys Res Commun 2006, 342:640-646

20. Kern J, Steurer M, Gastl G, Gunsilius E, Untergasser G: Vasohibin inhibits angiogenic sprouting in vitro and supports vascular maturation processes in vivo. BMC Cancer 2009, 9:284

21. Bjorndahl MA, Cao R, Burton JB, Brakenhielm E, Religa P, Galter D, Wu L, Cao Y: Vascular endothelial growth factor-A promotes peritumoral lymphangiogenesis and lymphatic metastasis. Cancer Res 2005, 65:9261-9268

22. Chang LK, Garcia Cardena G, Farnebo F, Fannon M, Chen EJ, Butterfield C, Moses MA, Mulligan RC, Folkman J, Kaipainen A: Dose-dependent response of FGF-2 for lymphangiogenesis. Proc Natl Acad Sci USA 2004, 101:11658-11663

23. Kozaki K, Miyaishi O, Tsukamoto T, Tatematsu Y, Hida T, Takahashi $\mathrm{T}$, Takahashi T: Establishment and characterization of a human lung cancer cell line NCl-H460-LNM35 with consistent lymphogenous metastasis via both subcutaneous and orthotopic propagation. Cancer Res 2000, 60:2535-2540

24. Schledzewski K, Falkowski M, Moldenhauer G, Metharom P, Kzhyshkowska J, Ganss R, Demory A, Falkowska-Hansen B, Kurzen H, Ugurel S, Geginat G, Arnold B, Goerdt S: Lymphatic endotheliumspecific hyaluronan receptor LYVE-1 is expressed by stabilin-1+. $\mathrm{F} 4 / 80+, \mathrm{CD} 11 \mathrm{~b}+$ macrophages in malignant tumours and wound healing tissue in vivo and in bone marrow cultures in vitro: implications for the assessment of lymphangiogenesis. J Pathol 2006, 209:67-77

25. Hirakawa S, Brown LF, Kodama S, Paavonen K, Alitalo K, Detmar M: VEGF-C-induced lymphangiogenesis in sentinel lymph nodes promotes tumor metastasis to distant sites. Blood 2007, 109:1010-1017

26. Harrell MI, Iritani BM, Ruddell A: Tumor-induced sentinel lymph node lymphangiogenesis and increased lymph flow precede melanoma metastasis. Am J Pathol 2007, 170:774-786

27. Hong YK, Shin JW, Detmar M: Development of the lymphatic vascular system: a mystery unravels. Dev Dyn 2004, 231:462-473

28. Hirakawa S, Kodama S, Kunstfeld R, Kajiya K, Brown LF, Detmar M VEGF-A induces tumor and sentinel lymph node lymphangiogenesis and promotes lymphatic metastasis. J Exp Med 2005, 201:1089-1099

29. Schoppmann SF, Birner P, Stockl J, Kalt R, Ullrich R, Caucig C, Kriehuber E, Nagy K, Alitalo K, Kerjaschki D: Tumor-associated macrophages express lymphatic endothelial growth factors and are related to peritumoral lymphangiogenesis. Am J Pathol 2002, 161:947-956

30. Cursiefen C, Chen L, Borges LP, Jackson D, Cao J, Radziejewski C, D'Amore PA, Dana MR, Wiegand SJ, Streilein JW: VEGF-A stimulates lymphangiogenesis and hemangiogenesis in inflammatory neovascularization via macrophage recruitment. J Clin Invest 2004, 113:1040-1050

31. Murakami M, Zheng Y, Hirashima M, Suda T, Morita Y, Ooehara J, Ema $\mathrm{H}$, Fong $\mathrm{GH}$, Shibuya M: VEGFR1 tyrosine kinase signaling promotes lymphangiogenesis as well as angiogenesis indirectly via macrophage recruitment. Arterioscler Thromb Vasc Biol 2008, 28:658-664

32. Padera TP, Kadambi A, di Tomaso E, Carreira CM, Brown EB, Boucher Y, Choi NC, Mathisen D, Wain J, Mark EJ, Munn LL, Jain RK: Lymphatic metastasis in the absence of functional intratumor lymphatics. Science 2002, 296:1883-1886

33. Casanovas O, Hicklin DJ, Bergers G, Hanahan D: Drug resistance by evasion of antiangiogenic targeting of VEGF signaling in late-stage pancreatic islet tumors. Cancer Cell 2005, 8:299-309

34. Bergers G, Hanahan D: Modes of resistance to anti-angiogenic therapy. Nat Rev Cancer 2008, 8:592-603

35. Hwang RF, Yokoi K, Bucana CD, Tsan R, Killion JJ, Evans DB, Fidler $\mathrm{IJ}$ : Inhibition of platelet-derived growth factor receptor phosphorylation by STI571 (Gleevec) reduces growth and metastasis of human pancreatic carcinoma in an orthotopic nude mouse model. Clin Cancer Res 2003, 9:6534-6544

36. Nissen LJ, Cao R, Hedlund EM, Wang Z, Zhao X, Wetterskog D, Funa K, Brakenhielm E, Cao Y: Angiogenic factors FGF2 and PDGF-BB synergistically promote murine tumor neovascularization and metastasis. J Clin Invest 2007, 117:2766-2777

37. He Y, Kozaki K, Karpanen T, Koshikawa K, Yla-Herttuala S, Takahashi $\mathrm{T}$, Alitalo K: Suppression of tumor lymphangiogenesis and lymph node metastasis by blocking vascular endothelial growth factor receptor 3 signaling. J Natl Cancer Inst 2002, 94:819-825 